Z. klin. Chem. u. klin. Biochem.

9. Jg., S. $356-360$, Juli 1971

\title{
Analyse der Wasserräume und des Lipidgehalts des menschlichen subcutanen Fettgewebes ${ }^{1}$ )
}

\author{
Von AnNeliese Englhardt, H. Liebermeister, Th. Reuter und K. Irmscher \\ Aus der 2. Mediqinischen Klinike der Universität (Direktor: Prof. Dr. K. Oberdisse) \\ und dem Diabetes-Forschungs-Institut an der Universität Düsseldorf (Direktor: Prof. Dr. K. Oberdisse)
}

(Eingegangen am 4. März 1971)

\begin{abstract}
An Proben aus menschlichem subcutanem Fettgewebe von 22 Normgewichtigen und 20 Adipösen wurden Gesamtwasser, extrazelluläres und intrazelluläres Wasser, sowie Lipide gemessen.

Die Bestimmung des Gesamtwasserraumes erfolgte als $\mathrm{H}_{2} \mathrm{O}-\mathrm{Raum}$, die des extrazellulären Wasserraums als Sorbit-Raum. Zur Messung wurden die markierten Substanzen $\mathrm{H}_{2} \mathrm{O}-\left[{ }^{3} \mathrm{H}\right]$ und Sorbit-[U-14 $\left.\mathrm{C}\right]$ verwendet. Die Fehlerquellen der analytischen Untersuchungen und die Reproduzierbarkeit der Werte wurden geprüft.

Für das intrazelluläre Wasser wurde ein Mittelwert von $53,9 \mathrm{ml} / \mathrm{kg}$ bei Normgewichtigen und ein Mittelwert von $52,2 \mathrm{ml} / \mathrm{kg}$ bei Adipösen gefunden. Der extrazelluläre Wassergehalt war im Mittel 139,2 ml/kg bzw. 144,0 ml/kg in zwei Kollektiven Normgewichtiger und 142,3 bzw. 143,9 ml/kg Fettgewebe in zwei Kollektiven Adipöser.

Gesamtwasserraum und Extrazellulärraum nahmen während einer zweistündigen Inkubationsperiode kontinuierlich zu.

Durch Zusatz von Insulin und Glucose zum Medium wurden die Werte für die Wasserräume nicht beeinflußt.

Der Lipidgehalt im Fettgewebe Adipöser war signifikant erhöht.
\end{abstract}

\section{Analysis of the water space and lipid concentration in buman subcutaneous adipose tissue}

Total water, extracellular and intracellular water and lipids were measured in samples of human subcutaneous adipose tissue from 22 persons of normal weight and 20 obese persons. Total water was determinded as the $\mathrm{H}_{2} \mathrm{O}$ space, and the extracellular water was measured as the sorbitol-[U-14 $\mathrm{C}]$ space. Sources of error in the analysis and the reproducibility of the values were tested.

In the persons of normal weight, the average intracellular water was $53.9 \mathrm{ml} / \mathrm{kg}$ adipose tissue and for obese persons $52.2 \mathrm{ml} / \mathrm{kg}$. The average extracellular water was $139.2 \mathrm{ml} / \mathrm{kg}$ and $144.0 \mathrm{ml} / \mathrm{kg}$ in two normal weight collectives, and 142.3 and $143.9 \mathrm{ml} / \mathrm{kg}$ adipose tissue in two obese collectives.

During a two hour incubation period, the total water and the extracellular space increased continually. The addition of insulin or glucose to the medium did not affect the values for the water spaces. The lipid content of adipose tissue from obese persons was significantly higher.

Untersuchungen der Zusammensetzung des menschlichen Fettgewebes führten bisher nicht zu einheitlichen Ergebnissen. Vor allem über den Wasser,- Protein- und Lipidgehalt liegen unterschiedliche Berichte vor $(1-5,12-16)$. Diese Differenzen werden von den einzelnen Autoren auf den wechselnden Anteil des Gewebes an unspezifischem Begleitgewebe zurückgeführt. Große individuelle Streuung von Stoffwechseldaten werden auf die Schwankungen dieser Bezugssysteme bezogen $(6,7,17)$. Bei Adipösen finden sich nach Angaben verschiedener Arbeitskreise besonders ausgeprägte Veränderungen dieser Bezugswerte, die die Reproduzierbarkeit vergleichender Messungen am Fettgewebe Normgewichtiger und Adipöser gefährden. Ursache ist vor allen Dingen eine von mehreren $\mathrm{Au}$ toren beschriebene Zunahme der Fettzellgröße bei Fettsüchtigen $(8,9)$. Chemische Zusammensetzung und Stoffwechselleistung des Fettgewebes ändern sich in Relation zur Fettzellgröße $(9-11,27)$. Andere Autoren berichten über Veränderungen des Wassergehalts im Fettgewebe Adipöser. Durch in vivo-Messungen wurde teilweise eine Verminderung, zum Teil auch eine Vergrößerung des extrazellulären Flüssigkeitsvolumens 1) Durchgeführt mit Unterstützung der Deutschen Forschungs-
gemeinschaft. gefunden $(12,13)$. Auch in vitro-Messungen führten nicht zu übereinstimmenden Ergebnissen.

Nachstehend wird über eine Methode berichtet, die eine Messung des Gesamtwassergehalts, des extrazellulären und intrazellulären Wassers des Fettgewebes in vitro ermöglicht. Mit dieser Methode wurden vergleichende Untersuchungen am subcutanen Fettgewebe Normgewichtiger und Adipöser in vitro durchgeführt. An denselben Gewebsproben wurde auch der Lipidgehalt gemessen.

\section{Methodik}

Patientengut und Gewinnung des Fettgewebes

Bei insgesamt 22 Normgewichtigen und 20 Adipösen wurden Proben von etwa $1 \mathrm{~g}$ Fettgewebe aus der oberen Schicht des subcutanen Fettgewebes der Abdominalregion entnommen. Die Excision erfolgte bei allen Normalgewichtigen und bei 15 Adipösen am Beginn chirurgischer Operationen, bei 5 Adipösen durch lokalen Eingriff in Anästhesie mit 0,5proz. Novocain. Patienten mit akut entzündlichen oder malignen Prozessen wurden von der Untersuchung ausgeschlossen. Alle Probanden standen einige Tage vor dem Eingriff in stationärer Beobachtung. Reduktionsdiäten wurden nicht verabreicht. Vor der Gewebsgewinnung wurden keine Salz- oder Glucosehaltigen Infusionslösungen infundiert. Nahrungskarenz vor dem Eingriff etwa $14 \mathrm{Stdn}$. Die Adipösen hatten ein Ubergewicht von $20-75 \%$. Alter des gesamten Kollektivs 19-45 Jahre. 


\title{
BIOCHIMIE
}

Edité par la Société de Chimie Biologique

\author{
tel est le titre \\ sous lequel paraîtra à partir de 1971 \\ le „BULLETIN DE LA SOCIÉTÉ DE CHIMIE BIOLOGIQUE“
}

\author{
SECRETARIAT \\ REDACTION \\ de la Société de Chimie Biologique \\ F. GROS, Secrétaire scientifique \\ F. PERCHERON, Secrétaire à la Publication \\ J. P. EBEL, Secrétaire Général (Relations Extérieures) \\ J. NUNEZ, Secrétaire à l'Information \\ R. PERLES, Secrétaire Général \\ Y. RAOUL, Secrétaire à l'Edition
}

SECRETARIAT et REDACTION: 4 Avenue de l'Observatoire, PARIS $6^{\circ}$

\section{FASCICULES}

ABONNEMENTS: FRANCE et ZONE FRANC: 150 ffres - BELGIQUE: $1.687,-$ frcs - AUTRES PAYS: $186,-$ ffres

MASSON et Cie, Editeurs - 120 Boulevard St Germain - PARIS 6ème

\section{Mikromethoden}

für das

\section{klinisch-chemische}

und

biochemische

Laboratorium



Walter de Gruyter

Berlin - New York

\section{Von Hermann Mattenheimer}

2., völlig neubearbeitete und erweiterte Auflage.

Oktav. Mit 35 Abbildungen. XVI, 223 Seiten. 1966.

Plastikeinband DM 30,-

Chemotechniker und medizinisch-chemische Assistenten erheben seit langem die Forderung, Untersuchungen geringster Blut- und Gewebeproben rasch und sicher durchzuführen. Der vorliegende Leitfaden wird dieser Aufgabe gerecht, da die erforderlichen Arbeitsvorschriften kurz, übersichtlich und leichtverständlich dargestellt sind.

Zeitschrift für analytische Chemie

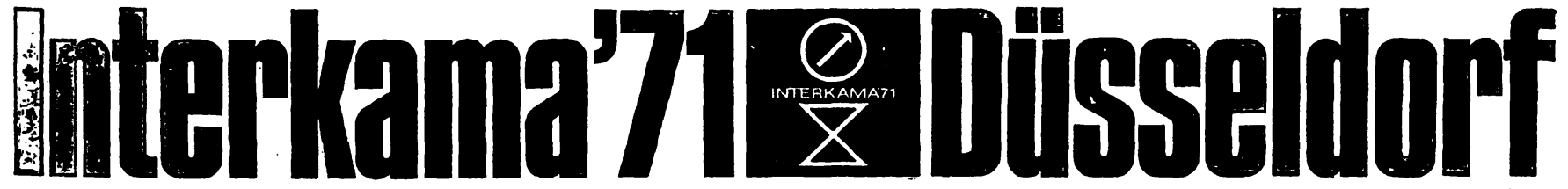

5. Internationaler Kongreß mit Ausstellung für Meßtechnik und Automatik vom 14. bis 20. Oktober 1971 Information: Dússeldorfer Messegeselischaft mbH - NOWEA- und Arbeltsgemeinschaft INTERKAMA, 4 Düsseldort, Dulsburger Str. 1a, Tel.: 02 11/4 4041 


\section{Autoradiography}

by

Dipl.-Chem. Helmut A. Fischer

Max-Planck-Institute for Brain Research, Neurochemical Research Group, Frankfurt/Main

and

Prof. Dr. Gottfried Werner

Max-Planck-Institute for Brain Research, Neurochemical Research Group, Frankfurt/Main

Octavo. X, 198 pages. With 93 figures and 14 tables. 1971. Bound DM 64, -; $\$ 18.80$

(Working Methods in Modern Science. Edited by Prof. Dr. Kurt Fischbeck)

"Autoradiography" describes the methods and techniques, possibilities and limitations, of the qualitative and quantitative autoradiography of macroscopic, microscopic and electron-microscopic specimens. The book is of interest to all those working with radioisotopes in scientific and medical fields and to all scientific and technical libraries, institutes, clinics and all laboratories of scientific subjects.

Weiterhin lieferbar:

Fischer-Werner, Autoradiographie. Mit 93 Abbildungen und 14 Tabellen. X, 214 Seiten. 1971. Gebunden DM 42, -


(Arbeitsmethoden der modernen Naturwissenschaften. Herausgegèben von Prof. Dr. Kurt Fischbeck).

\section{Walter de Gruyter - Berlin • New York}









\section{PLASTIBRAND-Wasserstrahlpumpe}

Erzeugnis einer Fabrik für Vakuumpumpen

Hohe Saugleistung

Rückschlagsicherheit auch bei niedrigem Wasserdruck

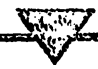

Keine Wassereinbrüche in die Apparatur beim Abstellen der Pumpe
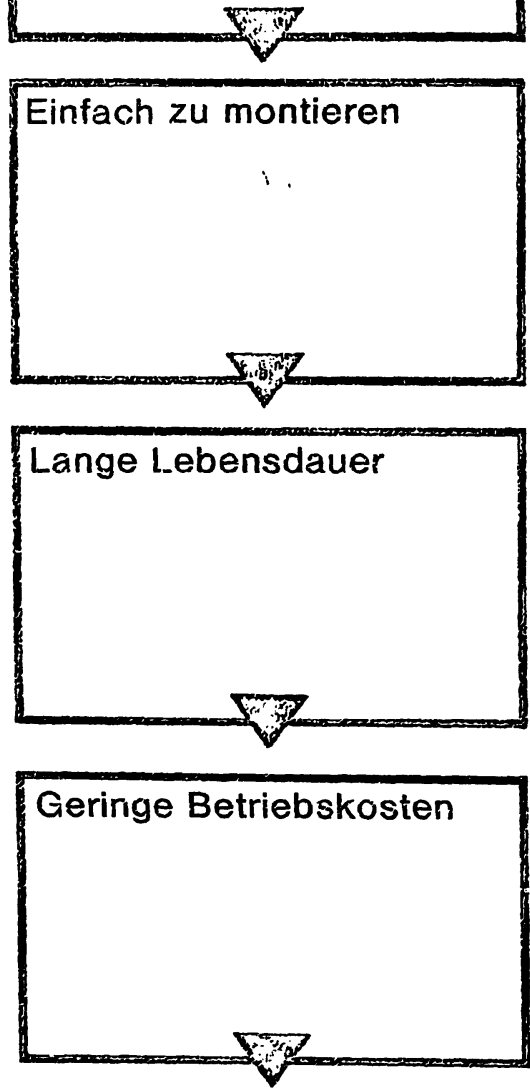

Die inneren Abmessungen einer Wasserstrahlpumpe kann man „ausprobieren” oder errechnen. Die Plastibrand-Pumpe ist errechnet

\section{Eingebautes}

Rückschlagventil

Gewindeanschlüsse 1/2", $3 / 8^{\prime \prime}, 3 / 4^{\prime \prime}$, M $22 \times 1$ oder

Schlauchtülle

Einfacher Aufbau aus wenigen, robusten

Polypropylenteilen

Niedriger

Wasserverbrauch durch sorgfältig abgestimmte Querschnittsverhältnisse der Düsen

Günstiger

Anschaffungspreis


\section{BRAND-GERÄTE \\ WEIL DIE \\ RENTABILITÄT \\ ENTSCHEIDET}

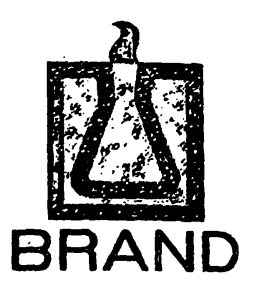




\section{Material und Reagenzien}

Zur Inkubation wurde ein modifizierter KREBS-RINGER-Bicarbonat-Puffer folgender Zusammensetzung verwendet:

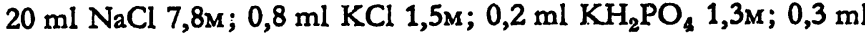
$\mathrm{CaCl}_{2} 1,37 \mathrm{M} ; 0,2 \mathrm{ml} \mathrm{MgSO}{ }_{4} 1,6 \mathrm{M}$; demin. Wasser ad $108 \mathrm{ml}$. Vor dem Gebrauch Zugabe von $21 \mathrm{ml} \mathrm{Na} \mathrm{CO}_{3}$ 0,12M. $20 \mathrm{Min}$. Durchströmung mit $\mathrm{O}_{2} / \mathrm{CO}_{2}(95 / 5 ; \nabla / v)$ Verwendung unter Gasphase, End-pH 7,4.

Human-Albumin reinst, Behring-Werke; gemessener Gehalt an freien Fettsäuren $6,7 \mu \mathrm{Mol} / \mathrm{g}$, gemessener Gehalt an Glycerin $0,55 \mu \mathrm{Mol} / \mathrm{g}$, elektrophoretischer Reinheitsgrad $100 \%$. Glucose pro analysi, reinst, für die Bakteriologie Merck. D-Sorbitol[U-14 C], vaccum sealed, $\mathrm{H}_{2} \mathrm{O}-\left[{ }^{3} \mathrm{H}\right]$ vaccum sealed, Radiochemical Centre, Amersham.

\section{Szintillationslösung}

0,13 g POPOP (1,4-Bis-2-(methyl-5-phenyl-oxazolyl)-benzol, Szintillationsgrad, Packard Comp.; 104,5 g Naphthalin (für Szintillationsmessungen, Merck AG) in $500 \mathrm{ml}$ Dioxan reinst, Merck, $500 \mathrm{ml}$ Toluol (p. a. Merck) und $300 \mathrm{ml}$ Methanol (p. a. Merck).

\section{Gewebsentnahme und Präparation}

Unmittelbar nach der Excision, die schonend unter Vermeidung von Zerreißungen des Gewebsverbandes durchgeführt wurde, wurden die Gewebsproben in einer feuchten Kammer in den Präparationsraum gebracht, der dem Operationssaal unmittelbar benachbart war. Dort wurden die Gewebsstücke mit zwei feinen Pinzetten durch Trennung der Bindegewebssepten in kleine Teile von etwa $20-30 \mathrm{mg}$ Gewicht zerteilt. Die Bindegewebsanteile wurden verworfen.

\section{Messung der Wasserräume}

Die Untersuchung der Wasserräume erfolgte unmittelbar nach der Präparation. Fettstückchen mit einem Gesamtgewicht von etwa $200-300 \mathrm{mg}$ wurden in Inkubationsgefäße mit einem Volumen von $2,5 \mathrm{ml}$ und einem Mediuminhalt von $0,5 \mathrm{ml}$ eingewogen. Zusammensetzung des Mediums: Krebs-Ringer-Bicarbonat-Puffer $\mathrm{pH} 7,4 \mathrm{mit} 0,2 \mu \mathrm{C}$ D-Sorbit-[U_- $\left.{ }^{14} \mathrm{C}\right]$ und $0,6 \mu \mathrm{C} \mathrm{H} \mathrm{H}_{2} \mathrm{O}-\left[{ }^{3} \mathrm{H}\right] / \mathrm{ml}$, $5 \mathrm{~g} / 100 \mathrm{ml}$ Human-Albumin, $100 \mathrm{mg} / 100 \mathrm{ml}$ Glucose, $50 \mathrm{mg} /$ $100 \mathrm{ml}$ Sorbit. Inkubation 20 bzw. 60 oder $120 \mathrm{Min}$. in der Warburg-Schüttelapparatur, Frequenz $80 \mathrm{Min}$., Temp. $37^{\circ}$. Nach Ende der Inkubation Entnahme von $2 \mathrm{mal} \mathrm{0,05} \mathrm{ml} \mathrm{Medium,} \mathrm{das}$ im Verbältnis 1:11 in 0,33M Perchlorsäure enteiweißt wurde. Der Rest des Mediums wurde im Inkubationsgefäß mit geschlossenem Deckel in trockenem $\mathrm{CO}_{2}$ eingefroren. Danach wurde die Wand des Probengefäßes in Höhe dex Oberfläche aufgeschnitten und der gefrorene Inbalt herausgenommen. Dieser wurde in gefrorenem

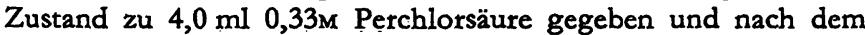
Auftauen $4 \mathrm{mal} 30 \mathrm{Sek}$. unter Eiskühlung homogenisiert (Ultraturrax). Das Homogenat wurde in der Ultrazentrifuge (Spinco L 50, Beckman Instruments) bei $2-4^{\circ}$ für 30 Min. bei $98000 \mathrm{~g}$ zentrifugiert, der klare Uberstand abgesaugt. Je $0,1 \mathrm{ml}$ des Úberstands bzw. des Perchlorsäureextrakts wurden zu $15 \mathrm{ml}$ Szintillationslösung gegeben. Die Messung der Isotope exfolgte im Tri-Carb-Flüssigkeits-Szintillationsspektrophotometer. Die Aktivitäten von ${ }^{14} \mathrm{C}$ und ${ }^{3} \mathrm{H}$ wurden nach einer Methode von TrAEGER (18) getrennt.

\section{Berechnungen}

Extrazellulärer Wasserraum (Sorbit-Raum).

Er errechnet sich aus den Impulstaten von ${ }^{14} \mathrm{C}$ in den Mediumproben vor und nach der Inkubation

$$
\frac{V_{M(m I)}}{G_{F(g)}} \cdot\left(\frac{c p m_{\text {to } M}}{c p m_{t E M}}-1\right)=\text { Sorbit-Raum 1/kg Fettgewebe }
$$

\section{Gesaminasserraum}

Er errechnet sich entweder aus den Aktivitäten von $\mathrm{H}_{2} \mathrm{O}-\left[{ }^{3} \mathrm{H}\right]$ vor und nach der Inkubation oder aus den Aktivitäten von Sorbit-[U_14 C], wenn die Aktivitäten auch im Homogenat gemessen wurden, da sich Sorbit nach dem Homogenisieren auch im gesamten Gewebswasser verteilt.

Formel für die Berechnung mit $\mathrm{H}_{2} \mathrm{O}-\left[{ }^{3} \mathrm{H}\right]$ :

$\frac{V_{\mathrm{M}(\mathrm{ml})}}{\mathrm{G}_{\mathrm{F}(\mathrm{g})}} \cdot\left(-\frac{\mathrm{cpm}_{\mathrm{to}} \mathrm{M}}{\mathrm{cpm} \mathrm{tEH}}-1\right)=\underset{\text { gewebe }}{\text { Gesamtwasserraum } 1 / \mathrm{kg} \text { Fett- }}$

\section{Intrazellulärer Wasserraum}

Das Volumen des intrazellulären Wasserraums ergibt sich aus den Impulsraten für ${ }^{14} \mathrm{C}$ des Mediums nach der Inkubation und denen des Extrakts nach folgender Formel

$\frac{V_{M(m)}}{G_{F(g)}} \cdot\left(\frac{c p m t E M}{c p m t E H}-1\right)=\underset{\text { Fettgewebe }}{\text { Intrazellulärer Wasserraum } 1 / k g}$

Der intrazelluläre Wasserraum kann auch aus der Differenz vom Gesamtwasserraum und Sorbitraum berechnet werden.

\section{Abkilrzungen}

$\mathrm{V}_{\mathbf{M}}$ Volumen des Inkubationsmediums (ml).

GF Gewicht des inkubierten Fettgewcbes (g).

to Beginn der Inkubationsperiode.

$t_{E}$ Ende der Inkubationsperiode.

cpm M Imp./Min. im Medium.

cpm H Imp./Min. im Extrakt.

\section{Gewebslipide}

Der Lipidgehalt wurde gleich dem Triglyceridgehalt gesetzt, da nach Angaben von HrRSCF und Mitarbeitern (7) $99 \%$ der Fettzellipide beim Menschen Triglyceride sind. Zur Triglyceridbestimmung wurde die Methode von EgGSTEIN und Kreutz, modifiziert von Jahnke und Herberg $(21,22)$ verwendet. Die Messung erfolgte ohne Extraktion im Homogenat mit alkalischer Verseifung der Triglyceride. Diese Methode war an Reproduzierbarkeit derjenigen nach Extraktion der Lipide überlegen. Verdünnung Fettgewebe: Triäthanolamin-HCl-Puffer $(50 \mathrm{mMol}+$ $5 \mathrm{mMol}$ EDTA) $1: 5$ bis $1: 10$. Homogenisiert wurde im Ultraturrax bei $0^{\circ} 4 \mathrm{mal} 30$ Sek. Bei der Umrechnung auf Gewichtseinheiten wurde ein empirisches Molekulargewicht von 885 zugrunde gelegt. Die Berechnung des Lipidvolumens erfolgte mit Hilfe des mittleren spezifischen Gewichts der menschlichen Fett-Zellipide nach Extraktion nach Folch (24).

\section{Statistische Berechnungen}

Berechnung der Signifikanz der Differenzen aller Parameter bei Normgewichtigen und Adipösen mit Hilfe des t-Tests. Der Fehlerinhalt einzelner Teilschritte am Gesamtfehler wurde durch Varianzanalyse ermittelt.

\section{Kommentar zur Methodik}

Fehlerquellen der Methode

Eine große Anzahl von Vorversuchen wurde durchgeführt, um die analytischen Verfahren zu vereinfachen und die Fehlermöglichkeiten zu verringern.

1. Erhebliche Fehler können durch den Transport der Fettgewebsproben vom Operationssaal zum Laboratorium entstehen: Alterung der Proben, Austrocknung oder Quellung. Das Austrocknen wurde durch Einbringen der Proben am Operationstisch in eine feuchte Kammer verhindert. Die Quellung wurde durch rasches Einbringen der Proben bereits im Operationsbereich in eine glucose- und eiweißhaltige Lösung auf das geringst mögliche $\mathrm{Ma} \beta$ reduziert. Aufbewahren der Proben bis zur Messung in einem salzhaltigen Medium ohne Glucose und Albumin führt zu einer schlechteren Reproduzierbarkeit der Ergebnisse (20) (Tab. 1). 
Fehler entstehen dadurch, daß am Ende der Inkubationsperiode das Gewebe in Perchlorsäure gebracht werden muß. Durch Einbringen der gesamten Probe (Gewebe + Medium) in gefrorenem Zustand in die Perchlorsäure wurde jede mögliche Übertragung von Isotopen, die

Tab. 1

Mittelwerte, Standardabweichung und Variationskoeffizienten der Messung des Extrazellulärraums des menschlichen subcutanen Fettgewebes mit verschiedenen Methoden

a) Aufbewahrung bis zur Inkubation in glucosefreiem Puffer, Präa) Aurbewach Spülen und Trocknen auf Filterpapier, Einwiegen der paration nach Spülen und Trocknen auf Filterpapier,
Fettstücke in ein glucosehaltiges Medium b) Siehe Methodik

c) Am Ende der Inkubation wird das Fettgewebe auf einem Plastiksieb gespült und dann in $0,33 \mathrm{M}$ Perchlorsäure gebracht

d) Siehe Methodik

Inkubationsdauer für $a-d 120 \mathrm{Min}$.

\begin{tabular}{|c|c|c|c|c|}
\hline & $\mathrm{n}$ & $\begin{array}{c}\overline{\mathbf{x}} \\
{[\mathrm{ml} / \mathrm{kg}}\end{array}$ & $\begin{array}{c}\mathbf{s} \\
\text { vebe] }\end{array}$ & V\% \\
\hline $\begin{array}{l}a \\
b \\
c \\
d\end{array}$ & $\begin{array}{r}12 \\
10 \\
6 \\
12\end{array}$ & $\begin{array}{l}242,0 \\
246,5 \\
263,2 \\
230,2\end{array}$ & $\begin{array}{l}64,0 \\
26,3 \\
55,0 \\
21,3\end{array}$ & $\begin{array}{r}26,4 \\
10,6 \\
20,9 \\
9,2\end{array}$ \\
\hline
\end{tabular}

dem Gewebe anhaften, vermieden. Der Isotopengehalt des Mediums wurde in der Berechnung berücksichtigt.

\section{Reproduzierbarkeit der Werte \\ Wasserräume}

Es mußte geprüft werden, ob markierter Sorbit ein geeigneter Indikator für die Messung des Extrazellulärraums im menschlichen Fettgewebe ist. Es konnte nachgewiesen werden, daß der Zucker im menschlichen Fettgewebe nicht metabolisiert wird.

Die ${ }^{14} \mathrm{CO}_{2}$-Bildung aus Sorbit-[U-14 $\left.\mathrm{C}\right]$ in Gegenwart von Fettzellproben konnte quantitativ vernachlässigt werden, da sie unter 0,1\% lag. Zu übereinstimmenden Ergebnissen kamen KaHLENBERG und KaLANT am Rattenfettgewebe (17). Messungen von CROFFord und RENolD am epidydimalen Fettgewebe der Ratte und von KaHLENBERG und KaLANT am menschlichen Fettgewebe zeigten übereinstimmend, $\mathrm{da} ß$ sich Sorbit besser zur Messung des extrazellulären Raums eignet als Saccharose $(17,19)$. Der Saccharose-Raum war nach KAHLENBERG und KaLANT gleich oder größer als der Gesamt-Wasserraum, so daß ein Eintrom von Saccharose in die Zellen angenommen werden muß. In eigenen Untersuchungen ergab die Messung des intrazellulären Wassers mit Sorbit-[U_14 C] aus dem Extrakt und durch Berechnung der Differenz Gesamt-Wasserraum Sorbit-Raum übereinstimmende Resultate. Sorbit-Raum und Gesamt-Wasserraum nehmen bei längerer Inkubation in gleichem Umfang zu, die Differenz bleibt konstant.

Die Richtigkeit der Werte wurde auch für den GesamtWasserraum geprüft durch Vergleich der Isotopenmethode mit der Bestimmung des Feucht- und Trokkengewichts. $\mathrm{Da}$ der Gesamt-Wasserraum während der Inkubation zunimmt, mußten die Werte für diesen Vergleich nach $t_{0}$ extrapoliert werden. Diese graphisch extrapolierten Werte zeigten gute Übereinstimmung mit denen der Differenzwägung.

\section{Lipide}

Bei der Untersuchung von Stichproben aus Fettgewebsextrakten ergaben sich zunächst stark variierende Werte. Durch Varianzanalyse konnte nachgewiesen werden, daß der Fehler im wesentlichen durch die Entnahme aliquoter Teile des Homogenats bedingt war, da die Lipide rasch zurr Oberfläche flottierten. Erst bei Entnahme unter kontinuierlichem Mischen wurden reproduzierbare Werte erhalten.

\section{Ergebnisse}

Wasserräume des Fettgenebes Normgewichtiger unter verschiedenen experimentellen Bedingungen (Tab. 2, 3, 4 und 5)

Die Werte für den Gesamtwasserraum wurden sowohl mit Sorbit-[U- $\left.{ }^{14} \mathrm{C}\right]$ als auch mit $\mathrm{H}_{2} \mathrm{O}-\left[{ }^{3} \mathrm{H}\right]$ gemessen. Die Werte stimmten überein mit denẹn, die aus dèr Differenz

Tab. 2

Wasserraum des menschlichen subcutanen Fettgewebes mit verschiedenen Methoden ermittelt Versuchsbedingungen siehe Methodik a) Bestimmung mit $\mathrm{H}_{2} \mathrm{O}-\left[{ }^{3} \mathrm{H}\right]$

b) Bestimmung mit Sorbit-[U-14 C] vor und nach dem Homogenisieren c) Differenzwägung vor und nach dem Trocknen bis zur Gewichtsdie Summe aus Lipiden und fettfreiem Trockengewicht des Restgewebes

Die Werte für a) und b) wurden bestimmt nach $20 \mathrm{Min}$. Aquilibrierung und aus weiteren Messungen bei $t_{0}, t_{80}$ und $t_{120}$ wurde durch $B e-$
rechnung der sich aus allen Einzelwerten ergebenden Gerade nach

\begin{tabular}{|c|c|c|c|}
\hline & $\mathrm{n}$ & \multicolumn{2}{|c|}{ [ml/kg Fettgewebe] } \\
\hline $\begin{array}{l}a \\
b \\
c\end{array}$ & $\begin{array}{r}12 \\
10 \\
5\end{array}$ & $\begin{array}{l}193,1 \\
182,7 \\
179,5\end{array}$ & $\begin{array}{l}20,3 \\
24,8 \\
40,5\end{array}$ \\
\hline
\end{tabular}

Tab. 3

Extrazelluläre Flüssigkeitsräume und intrazellulärer Wassergehalt im menschlichen subcutanen Fettgewebe, gemessen nach verschiedenen Zeiten der Vorinkubation. Absolutwerte $\bar{x} \pm s$ aus 20 Experimenten (Fettgewebsproben von 10 Normgewichtigen und 10 Adipösen). Die $t_{0}$-Werte für die extrazellulären Räume (Sorbit-Raum) wurden durch graphische Extrapolation nach $t_{0}$ gewonnen, die Werte für die Intrazellulärräume berechnet aus der Differenz Gesamt-Wasserraum Sorbit-Raum

\begin{tabular}{lrrrr}
\hline & \multicolumn{1}{c}{$t_{0}$} & \multicolumn{1}{c}{$t_{20}$} & $t_{60}$ & $t_{120}$ \\
& Normgewichtige & & \\
\hline Sorbit-Raum [ml/kg] & 140,0 & 157,4 & 190,3 & 236,1 \\
Intrazellulär-Raum & $\pm 16,7$ & $\pm 17,1$ & $\pm 24,3$ & $\pm 19,3$ \\
[ml/kg] & 50,3 & 51,2 & 54,3 & 53,2 \\
& $\pm 12,7$ & $\pm 13,8$ & $\pm 8,9$ & $\pm 15,3$ \\
Sorbit-Raum [ml/kg] & Adipöse & & \\
& 147,3 & 162,7 & 187,2 & $\mathbf{2 2 7 , 5}$ \\
Intrazellulär-Raum & $\pm 53,7$ & $\pm 16,9$ & $\pm 20,9$ & $\pm 25,8$ \\
[ml/kg] & 53,7 & 54,1 & 53,7 & 56,2 \\
& $\pm 6,9$ & $\pm 8,9$ & $\pm 12,1$ & $\pm 13,2$ \\
\hline
\end{tabular}

Feuchtgewicht - (Gesamtlipide + fettfreies Trockengewicht) erhalten wurden. Auch die mit ${ }^{14} \mathrm{C}$ und ${ }^{3} \mathrm{H}$ gemessenen Werte waren nicht signifikant unterschiedlich. Durch Zusatz von steigenden Glucosekonzentrationen und Insulin $(1000 \mu \mathrm{E} / \mathrm{ml})$ wurden die Werte für Gesamtwasser-, Sorbit- und intrazellulären Raum, auch bei längerer Inkubation (120 Min.) nicht beeinflußt. Im inkubierten Gewebe stieg der Gesamtwassergehalt und der extrazelluläre Wasserraum in Relation zur Inkubationsdauer. Der Anștieg des ge- 


\title{
Disc Electrophoresis
}

and Related Techniques of Polyacrylamide Gel Electrophoresis

\section{by Dr. rer. nat. H. Rainer Maurer}

Max-Planck-Institut für Virus-Forschung Tübingen, Germany

Second revised and expanded edition

XVI, 224 pages. With 88 figures, 16 tables and 948 literature references. 1971

Bound DM 68, -; $\$ 19.75$

(Working Methods in Modern Science. Edited by Prof. Dr. K. Fischbeck)

\begin{abstract}
This updated and revised monograph in English language brings together — as far as possible — the body of widely dispersed knowledge and experience obtained from methods in disc electrophoresis and related techniques of polyacrylamide gel electrophoresis. The book is intended to be a laboratory manual with the purpose of sparing the user time consuming literature study and technical errors as much as possible. Included are exact procedures for the most varied separation problems, a survey of types of apparatus, descriptions of methods for detection and quantitation of proteins, nucleic acids, enzymes, antigens and radioactivities, a detailed treatment of micro disc electrophoresis, preparative polyacrylamide gel electrophoresis and many other techniques for special problems. In addition, this monograph also cites examples of results from research in biochemistry, clinical chemistry and food technology with the intention of offering suggestions and showing the versatility of the method. From the information in this book, the user should be able, without any previous experience, to solve his separation problems to his (her) satisfaction.
\end{abstract}

\section{Table of Contents}

Theoretical Background: The Polyacrylamide Gel-Important Principles of Electrophoresis-Theory of Disc Electrophoresis-Polyacrylamide Gel Electrophoresis with Discontinuous Voltage Gradients -

Method of Analytical Disc Electrophoresis: Physiochemical Properties of the Gel Components and their Identification-Equipment for Disc Electrophoresis-Procedures-Disc Enzyme Electrophoresis-Disc Immunoelectrophoresis

Micro-Disc Electrophoresis: General Methods-Micro-Disc Electrophoresis combined with Micro-Enzymeand Immuno-Analysis

Preparative Polyacrylamide Gel Electrophoresis: General Comments-Methods of Preparative Disc Electrophoresis

Applications in Clinical Chemistry: Blood Proteins - Proteins of other Body Fluids - Tissue Enzymes Other Tissue Proteins - Epidermal, Hair and Nail Proteins

Biochemical Applications: Enzymology - Endocrinology - Immunology - Animal Body Fluids Neurology - Entomology - Botany - Microbiology - Nucleic Acids

Applications in Food Technology and Forensic Analysis: Note - List of Abbreviations - References List of firms (manufacturers) - Index - Fold-out chart: Brief instructions for the preparation of gels and the electrophoretic procedure - Useful gel systems for disc electrophoresis

\section{Weiterhin lieferbar:}

H. R. MAURER, Disk-Elektrophorese. Theorie und Praxis der diskontinuierlichen PolyacrylamidgelElektrophorese. Mit èinem Geleitwort von E. Hecker. Mit 82 Abbildungen, 15 Tabellen, 1 Ausschlagtafel, 578 Literaturangaben. XVI, 221 Seiten. 1968. Geb. DM 36, -

(Arbeitsmethoden der modernen Naturwissenschaften. Herausgegeben von Prof. Dr. K. Fischbeck)

\section{Walter de Gruyter • Berlin • New York}




\section{Wenn Sie von einem Rührer \\ Es liegt vor:}

spitzenleistun-

gen erwarten, dürfen wir Sie sicher bald

\section{zul unseren} zuffritedemen Kunden zäliten.

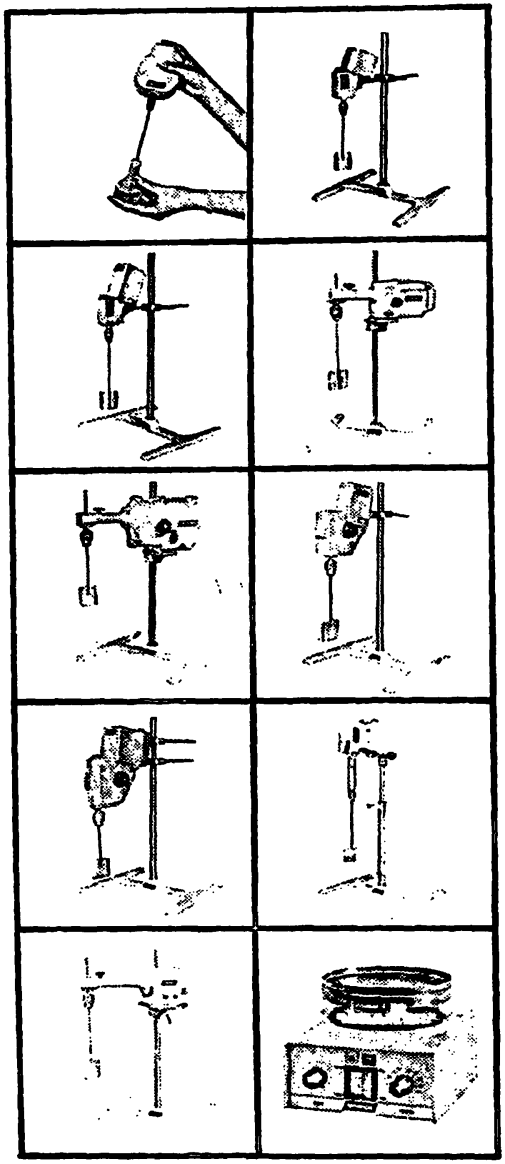

Heidolph-Rührer erzielen nämlich, dank ihrer ausgereiften Technik, absolute Spitzenleistungen im Laborbetrieb. Eine breite Palette, vom universell einsetzbaren Handrührer bis zum leistungsstarken Labor- und Produktionsrührer oder zum Magnetrührer, bietet für alle Erfordernisse das richtige Gerät. Bitte fordern Sie Prospektmaterial an.

Unser Fertigungsprogramm umfaßt außerdem: Verdampfer, Pumpen, Misch- und Schüttelgeräte, Spaltpol- und Kondensatormotore, Stirnradund Schneckengetriebe, stufenlos regelbare Getriebe, Gebläse. Einen ausführlichen Katalog halten wir für Sie bereit.

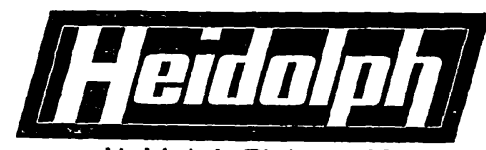

Heidolph-Elektro KG

D 8420 Kelheim/Germany

Tel. 09441 / 75 33, Telex $065415 d$

\section{Buddecke}

\section{Grundriß der Biochemie}

\author{
für Studierende der Medizin, \\ Zahnmedizin und Naturwissenschaften
}

Von Prof. Dr. Eckhart Buddecke

2. Auflage

Mit mehr als 400 Formeln, Tabellen und Diagrammen

XXXI, 499 Seiten. 1971. Plastik flexibel DM 27,50

Die progressive Zunahme des biochemischen Fachwissens erfordert eine überschaubare und zusammenfassende Darstellung der Biochemie als Hilfsmittel für den Unterricht. Der Grundriß der Biochemie von E. Buddecke gliedert den Wissensstoff in die Kapitel „Stoffe und Stoffwechsel", ,Stoffwechselregulation" und „Funktionelle Biochemie der Organe und Gewebe" mit dem Ziel, durch knappe Darstellung gesicherter Fakten und gezielte Stoffauswahl dem Leser einerseits eine rasche Information zu bieten, andererseits jedoch auf die vielfältigen Beziehungen und Anwendungsmöglichkeiten der Biochemie zur klinischen Chemie und Molekularpathologie hinzuweisen, um damit dem Studietrenden der Medizin und Zahnmedizin sowie dẹr anderen Gebiete der Naturwissenschaften das Verständnis klinischen Fachwissens zu erleichtern.

Stoffe und Stoffwechsel

Inhaltsübersicht

Chemische Zusammensetzung und Stoffwechsel der Zelle - Der Stoffwechsel als chemische Reaktionsfolge - Enzyme - Coenzyme - Aminosäuren - Nucleinsäuren - Proteine - Glucose und Kohlenhydrate - Lipide - Citratzyklus und biologische Oxydation - Porphyrine - Wasserhaushalt Mineralhaushalt

\section{Stoffwechselregulation}

Selbstregulation durch Rückkopplung - Regulation durch Metabolitkonzentrationen - Enzymkonkurrenz - Hormone

Einführung · Glanduläre Hormone · Gewebshormone

\section{Vitamine}

Definition und Klassifizierung - Vitamine mit Coenzymfunktion - Vitamine ohne Coenzymfunktion - Vitaminähnliche Wirkstoffe

\section{Funktionelle Biochemie der Organe und Gewebe}

Biochemie der Zelle - Blut - Leber - Verdauung und Resorption - Niere und Urin - Muskel Nervengewebe - Binde- und Stützgewebe - Wachstum und Abwehr

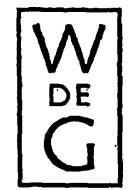


Tab. 4

Gesamtwasserräume (GWR), extrazelluläre Wasserräume (SR) und intrazellulärer Wassergehalt (IZR) im menschlichen Fettgewebe bei steigenden Glucosekonzentrationen im Medium mit und ohne Insulin. Mittelwerte $\bar{x} \pm s$ von 12 Normgewichtigen. Um die Ånderungen der Wasserräume bei längerer Inkubationszeit zu prüfen, wurden die Messungen nach $120 \mathrm{Min}$. vorgenommen

GWR Gesamt-Wasserraum SR Sorbit-Raum IZR Intrazellulär-Raum

\begin{tabular}{|c|c|c|c|c|}
\hline$\underset{\text { [mM] }}{\text { Glucose }}$ & $\begin{array}{l}\text { Insulin } \\
{[1}\end{array}$ & GWR & $\begin{array}{c}\text { SR } \\
{[\mathrm{ml} / \mathrm{kg} \text { Fettgewebe] }} \\
(\overline{\mathbf{x}} \pm \mathrm{s})\end{array}$ & IZR \\
\hline 2,8 & $\cdot-$ & \multirow{8}{*}{$\begin{array}{r}283,3 \\
\pm 33,2 \\
293,2 \\
\pm 19,8 \\
300,2 \\
\pm 30,8 \\
293,4 \\
\pm 31,7 \\
282,0 \\
\pm 29,5 \\
302,9 \\
\pm 39,8 \\
290,3 \\
\pm 38,9 \\
296,0 \\
\pm 24,0\end{array}$} & \multirow{8}{*}{$\begin{array}{r}230,2 \\
\pm 21,3 \\
250,9 \\
\pm 20,6 \\
293,4 \\
\pm 31,7 \\
237,6 \\
\pm 36,5 \\
232,2 \\
\pm 25,2 \\
248,1 \\
\pm 30,8 \\
245,9 \\
\pm 30,7 \\
246,9 \\
\pm 30,9\end{array}$} & \multirow{8}{*}{$\begin{array}{r}53,1 \\
\pm 12,7 \\
44,0 \\
\pm 14,2 \\
55,6 \\
\pm 16,2 \\
58,2 \\
\pm 10,4 \\
54,8 \\
\pm 13,9 \\
49,9 \\
\pm 14,6 \\
54,0 \\
\pm 14,2 \\
52,3 \\
\pm 12,2\end{array}$} \\
\hline 5,6 & - & & & \\
\hline 5,6 & + & & & \\
\hline 11 & - & & & \\
\hline 11 & + & & & \\
\hline 22 & - & & & \\
\hline 22 & - & & & \\
\hline 33 & - & & & \\
\hline
\end{tabular}

Tab. 5

Zusammensetzung des subcutanen menschlichen Fettgewebes Norm-

Mittelwerte von je 12 Newichtiger und Adipöser Mittelwerte von je 12 Normgewichtigen und 10 Adipösen. Die Gesamtwasserräume wurden mit $\mathrm{H}_{2} \mathrm{O}-\left[{ }^{3} \mathrm{H}\right]$ gemessen, die extrazellulären Wasserräume mit D-Sorbit-[U-'AC]; die intrazellulären Räume wurden berechnet aus der Differenz Gesamtwasserraum - Sorbit-Raum Die fettfreie Restsubstanz wurde berechnet aus der Differenz GeDie fettfreie Restsubstanz wurde berechnet aus der Differenz Ge-
samtvolumen Berechnung des Lipidvolumens wurden die Gewichtseinheiten des
Fettgewebes in Volumeneinheiten mit Hilfe des spez. Gewichtes um-
gerechnet

Da für diese Berechnungen die Mittelwerte der Kollektive verwendet wurden, können. Werte für s nicht angegeben werden

Für die Ermittlung der spez. Gewichts wurden Fettgewebsproben Für die Ermittlung der spez. Gewichts wurden Fettgev

Für die Fettgewebslipide ergab die statistische Berechnung für Normgewichtige/Adipöse $\mathrm{p}<0,05$

\begin{tabular}{lccccc}
\hline & Lipide & $\begin{array}{c}\text { Gesamt- } \\
\text { Wasser- } \\
\text { Raum } \\
\text { [ml/kg Fettgewebe] }\end{array}$ & $\begin{array}{c}\text { Sorbit- } \\
\text { Raum }\end{array}$ & $\begin{array}{c}\text { Intra- } \\
\text { zellulär- } \\
\text { Raum }\end{array}$ & $\begin{array}{c}\text { Fett- } \\
\text { freier } \\
\text { Raum } \\
\text { ml/kg }\end{array}$ \\
\hline Normge- & $\mathbf{7 5 7 , 0}$ & 193,1 & 139,2 & 53,9 & 89,9 \\
wichtige & $\pm 94,4$ & $\pm 20,3$ & $\pm 29,1$ & $\pm 11,5$ & 40,0 \\
Adipöse & $\mathbf{8 0 3 , 9}$ & 196,1 & 143,9 & 52,2 & 40,0 \\
& $\pm 89,0$ & $\pm 26,5$ & $\pm 21,3$ & $\pm 9,3$ & \\
\hline
\end{tabular}

samten Gewebswassers ist demnach durch Vergrößerung des extrazellulären Raums bedingt. $\mathrm{Da}$ sich ein Gleichgewicht zwischen Medium und Extrazellulärraum erst nach einer Inkubationsdauer von $15-20 \mathrm{Min}$. einstellt, mußten die Werte nach $t_{0}$ extrapoliert werden. Mit dieser Methode wurde bei Normgewichtigen ein mittlerer Extrazellulärraum von $144,0 \pm 16,7 \mathrm{ml} / \mathrm{kg}$ Fettgewebe gefunden, d. i. 14,1 Vol\% des Fettgewebes, wenn man das spezifische Gewicht des Gewebes berücksichtigt.

\section{Wasserräume im Fettgewebe Adipöser}

In weiteren Untersuchungen wurden die Wasserräume des Fettgewebes Adipöser mit demjenigen Normgewichtiger verglichen. Für jedes Gewebe wurden die Werte für den Gesamtwasserraum als $\mathrm{H}_{2} \mathrm{O}-\left[{ }^{3} \mathrm{H}\right]$-Raum und für den Sorbitraum als Sorbit-[U-14C]-Raum sowie deren Differenz errechnet. Signifikante Differenzen der Wasserräume Normgewichtiger und Adipöser waren nicht nachweisbar (Tab. 5).

\section{Lipidgebalt des Fettgewebes Normgewichtiger und Adipöser}

Der Lipidgehalt des Fettgewebes Adipöser war gering größer als derjenige bei Normgewichtigen. Die Unterschiede waren aber im t-Test signifikant. Berechnet man nun aus diesen Werten durch Subtraktion Gewebsvolumen - (Gesamtwassergehalt + Gesamtlipidgehalt) den Restraum, der aus fettfreier, wasserfreier Trockensubstanz bestehen muß, so findet sich ein Wert von $91,0 \mathrm{ml} / \mathrm{kg}$ für Normgewichtige, dagegen von nur 40,0 ml $/ \mathrm{kg}$ für Adipöse (Tab. 5).

\section{Diskussion}

Die Analyse zahlreicher Funktionen des Fettgewebes ist abhängig von der Größe der Flüssigkeitsräume. Beispielsweise ist zur Analyse der Kinetik von Transportprozessen die Kenntnis von der Größe des extrazellulären Raums notwendig, in den die Diffusion stattfindet. Auch die Messung extra- und intrazellulärer Konzentrationen bestimmter Metabolite ist nur möglich bei Kenntnis der Größe des extra- und intrazellulären Raums. Die gleichzeitige Bestimmung dieser Räume kann auf einfache Weise durch Verwendung von zwei Substanzen durchgeführt werden, von denen sich die eine im gesamten Gewebe, die andere lediglich im extrazellulären Raum verteilt. Markierte Substanzen müssen bei simultaner Messung durch besondere' Verfahren getrennt werden.

Sorbit ist unter den gewählten Versuchsbedingungen geeignet als Indikator des extrazellulären Raums im Fettgewebe. Er verteilt sich rasch zwischen Medium und extrazellulärer Flüssigkeit; das Gleichgewicht wird weder durch Insulin noch durch Glucose beeinflußt. Die Oxydation im Gewebe ist zu vernachlässigen. Ein Eindringen in die Zelle ist nach den vorliegenden Ergebnissen auszuschließen.

Die von uns ermittelten Werte für den extrazellulären Raum liegen im Bereich der von anderen Untersuchern ermittelten Werte. Gabe, Irmscher und HaEfs ermittelten auf indirektem Weg durch in vivo-Messungen (13) einen Wert von $15 \%$, MORSE und SOELDNER (14) einen solchen von $16,5 \%$ mit indirekten Methoden.

KaHLENBerg und KaLANT (17) fanden am omentalen Fettgewebe einen Sorbit-Raum von $16,3 \pm 2,6 \%$ bei einer Inkubationsperiode von 20 Min. Dieser Wert stimmt mit unserem bei gleicher Inkubationsperiode gefundenen Wert überein, wurde allerdings von den Autoren nicht extrapoliert. Dagegen fanden die Autoren durch Differenzwägung einen Gesamtwasserraum von $40,4 \pm 6,0 \%\left(\overline{\mathbf{x}} \pm s_{\bar{x}}\right)$ und kommen damit $z u$ einer Relation von intra- zu extrazellulärem Wasser von 1,5:1. In eigenen Untersuchungen erhielten wir für den Intrazellulärraum bei $t_{20}$ einen Wert von $51,2 \pm 13,8 \mathrm{ml} / \mathrm{kg}$, d. i. etwa $5 \%$ und damit eine Relation von intra- zu extrazellulärem Wasser von $1: 3$. Die Relation stimmt mit der von CROFFord und RENOLD am Rattenfettgewebe gemessenen überein (19). Dies würde bedeuten, daß im menschlichen Fett- 
gewebe ebenso wie im Rattenfettgewebe intrazelluläre Konzentrationen gegen einen hohen „background" extrazellulärer Gehalte gemessen werden.

Es fand sich kein Anhalt dafür, daß bei Fettsüchtigen ein veränderter Wassergehalt des Fettgewebes vorliegt. Andere Autoren berichten über erhöhte Wasserfraktionen bei Fettsucht $(3,29)$. Dagegen fand THomas (5) niedrigere Werte bei Personen mit 90-160\% Ubergewicht. Angaben nach in vivo-Bestimmungen mit Inulin, (Thiosulfat, korrigiert auf Inulin) ergaben ein z. T. wesentlich niedrigeres Extrazellulärvolumen von $6 \%$ (30) und $7 \%$ (31), lediglich KEYs und BROZEK geben mit Thiocyanat einen extrazellulären Anteil von $14 \%$ an (32), andere Autoren sogar Werte von 16,8 bis $19 \%$. GABE und IRMSCHER (26) wiesen aber darauf hin, $\mathrm{da} B$ das Verteilungsvolumen des Thiocyanat über dem des Inulin liegt, sie selbst fanden mit Inulin keine signifikanten Unterschiede des relativen Extrazellulärvolumens des Fettgewebes Fettsüchtiger und Normgewichtiger.

Die Variabilität der Resultate kann als Beweis der erheblichen methodischen Fehlerquellen dieser Messungen angesehen werden. Fehlerquellen der in vivoMessung sind, z. B. verschiedene Hydrierungsgrade unter exogenen Einflüssen. Auf die Irrtumsmöglichkeiten der in vitro-Messungen wurde in dieser Arbeit ausführlich eingegangen (z. B. Alterung, Quellung, Mediumwechsel).

Nimmt man an, daß die eigenen Ergebnisse in vitro den in vivo Werte nahe kommen, so ist im Fettgewebe Adipöser der extrazelluläre Wassergehalt unverändert. Durch die Zunahme des Fettgewebes, dessen Gewicht auf das Doppelte bis Dreifache ánsteigen kann, muß sich auch der absolute Wert für diesen Raum erheblich vergrößern, der gesamte extrazelluläre Verteilungsraum nimmt damit $z u$.

Als weitere Beobachtung fand sich im Fettgewebe Fettsüchtiger ein erhöhter Lipidgehalt, als dessen Folge eine Reduktion des lipidfreien Restraums angenommen werden muß. Bei gleichbleibendem Wasserraum muß daher eine Abnahme der fettfreien plasmatischen Substanz angenommen werden. Beobachtungen über verminderte Enzymaktivitäten (23, 27) und Stoffwechselleistungen (28) des Fettgewebes Fettsüchtiger könnten darin eine Erklärung finden.

Den Ärzten der Chirurgischen Klinik der Universität Düsseldorf danken wir für die Hilfe bei der Gewinnung von Gewebsmaterial.

\section{Literatur}

1. Forbes, R. M., A. R. Cooper und H. H. Mrtchelt, J. biol. Chemistry 213, 359 (1953). - 2. Mirchell, H. H., T. S. Hamilton, F. R. Steggerda und H. W. BeAn, J. biol. Chemistry 158, 625 (1945). - 3. Pawan, G. L. S. und M. Clode, Biochem. J. 74, 90 (1960). - 4. Entenmian, C., W. H. Goldwater, N. S. Ayres und A. L. Behnke, J. Appl. Physiol. Wash. 13, 129 (1958). - 5. Thosas, L. W., Quart. J. Exper. Physiol., London 47, 179 (1962). 6. Gellmorn, A. und P. A. Marks, J. Clin. Invest. 48, 925 (1961). 7. Hrrsch, J. und B. Goldrick, Handbook of Physiol., Section 5. Adipose tissue Washington (1965). - 8. Bjurulf, P., Acta med. Scand., Suppl. 349, 7 (1959). - 9. Prerss, H., CH. Hesse-WortmanN, F. A. Gries und U. Jahnke, Vortragstagung Dtsch. Ges. Fettwiss., Mainz 1967. - 10. Faulhaber, I. D., H. H. Ditschuneit, H. Ditschunert und E. F. Pfeiffer, 4. Kongr. Dtsch. Diabetesgesellschaft, Ulm 1969. - 11. Dirschunert, H., I. D. Faulhaber und H. Ditschuneit, 4. Kongreß Disch. Diabetesgesellschaft. Ulm 1969. - 12. BANSI, H. W. und J. M. OlsEN, Acta endocr. K'hvn. 32, 113 (1959). - 13. GABE, D. und K. IrMSCHER, Klin. Wschr. 45, 701 (1967). - 14. MORse, W. T. und S. SOELDNER, Handbook of Physiol., Adipose tissueWashington(1965). 15. Bozenraad, O., Dtsch. Arch. klin. Med. 103, 120 (1911). 16. Allen, Th., H. J. KrzYwicki und I. E. Roberts, J. Appl. Physiol., Wash. 14, 1005 (1959). - 17. KahLENBerg, K. und N.
Kalant, Canad. J. Biochem. 42, 1623 (1964). - 18. Tragger, G., Atompraxis 11, 7 (1965). - 19. Crofford, O. B. und A. E. Renold, J. biol. Chemistry 240, 14 (1965). - 20. Englhardr, A., unveröffentlicht. - 21. JaHnKE, K. und M. Heriberg, Ätztl. Laborat. 15, 201 (1969). - 22. EgGsteIN, M. und F. H. Kreutz, Klin. Wschr. 44, 262 (1966). - 23. EnglhardT, A., F. A. Gries, H. LIEBermetster und K. JahnNke, Diabetologia (in Druck). 24. Folch, J. M., H. G. Lees und S. SloAne, J. biol. Chemistry 225, 497 (1957). - 15. Crofford, O. B. und Mitarbeiter, Helvet. physiol. pharmacol. acta 24, 45 (1966). - 26. GABE, D., K. IRMSCHER und E. LOHR, Klin. Wschr. 45, 711 (1967). - 27. ENGLhardt, A., J. Feld, F. A. Gries, H. Preiss und K. Jahinke, Verh. Dtsch. Ges. inn. Med., 75. Bd. J. F. Bergmann Verlag München 1969, S. 881. - 28. Englhardt, A., R. KaspareK, H. LIEBERMEISTER und K. JAHNKE, Hormon- und Stoffwechselforschung (in Druck). - 29. VAGUE, J. und GarRigues I. C., zit. nach Morse, W. I. und I. S. Soeldner, Handbook of Physiology, Adipose tissue Washington (1965). - 30. Joknston, L. C. und J. M. Bernstern, J. Laborat. Clin. Med., S. Louis 45, 109 (1955). - 31. LJungGreen, H., D. Ikkos und B. LuFi, Acta endocr., K'hrn. 25, 167 (1957). - 32. KEYY, A. und J. BrožEK, Physiol. Rev. 33,245 , (1953).
Prof. Dr. Anneliese Englhardt $355 \mathrm{Marburg} / \mathrm{Lahn}$ Emil-Mannkopff-Str. 1 